\title{
O ESCORPIONISMO EM PONTA GROSSA, PARANÁ, E O CONHECIMENTO DA POPULAÇÃO ACERCA DESSE AGRAVO
}

\section{THE SCORPIONISM IN PONTA GROSSA, PARANÁ, AND THE KNOWLEDGE OF THE POPULATION ABOUT THIS HAZARD}

\author{
Bianca Mayara KOTVISKI'; Emanuel Marques da SILVA²; Ana Carolina Pinto da \\ CRUZ $^{3}$; Gabriela Ferreira de SOUZA ${ }^{4}$; Rosilda Aparecida KOVALICZN \\ Regina Aranha RIBEIRO'; Tayanghi Karina LOS$^{7}$; Gisélia Burigo Guimarães \\ RUBIO $^{8}$; Ivana de Freitas BARBOLA ${ }^{9}$
}

${ }^{1}$ Mestranda em Ecologia e Conservação de Recursos Naturais pela Universidade Federal de Uberlândia; Bacharel em Ciências Biológicas pela Universidade Estadual de Ponta Grossa -UEPG; E-mail: bianca_kotviski@hotmail.com

${ }^{2}$ Mestre em Saúde Pública pela Fundação Oswaldo Cruz - FIOCRUZ; Biólogo da Divisão de Vigilância de Zoonoses e Intoxicações - DVVZI, Secretaria de Estado da Saúde do Paraná - SESA; E-mail: ems@sesa.pr.gov.br

${ }^{3}$ Bacharel em Ciências Biológicas pela Universidade Estadual de Ponta Grossa - UEPG; E-mail: ana.carol.cruz@hotmail.com

${ }^{4}$ Mestre em Agroecologia e Desenvolvimento Rural pela Universidade Federal de São Carlos; Licenciada em Ciências Biológicas pela Universidade Estadual de Ponta Grossa UEPG. E-mail: gabygaya.bio@hotmail.com

${ }^{5}$ Mestre em Educação pela Universidade Estadual de Ponta Grossa - UEPG; Professora Assistente, Departamento de Biologia Geral, Universidade Estadual de Ponta Grossa UEPG; E-mail: rosildak@uol.com.br

${ }^{6}$ Doutora em Ciências Geodésicas pela Universidade Federal do Paraná - UFPR; Professora Adjunta, Departamento de Geociências, Universidade Estadual de Ponta Grossa - UEPG; E-mail: selmar.aranha@gmail.com

${ }^{7}$ Licenciada em Ciências Biológicas pela Universidade Estadual de Ponta Grossa - UEPG; E-mail: tayanghi@hotmail.com

${ }^{8}$ Bacharel e Licenciada em Ciências Biológicas pela Pontifícia Universidade Católica do Paraná - PUCPR; Chefe da Divisão de Vigilância de Zoonoses e Intoxicações - DVVZI, Secretaria de Estado da Saúde do Paraná - SESA; E-mail: giseliarubio@sesa.pr.gov.br

${ }^{9}$ Autor para contato: Doutora em Ciências Biológicas (Entomologia) pela Universidade Federal do Paraná - UFPR; Professora Associada, Departamento de Biologia Geral, Universidade Estadual de Ponta Grossa - UEPG; Endereço: Av. Carlos Cavalcanti, 4748, CEP 84030-900 - Uvaranas, Ponta Grossa, Paraná, Brasil; E-mail: ibarbola@yahoo.com.br

\section{RESUMO}

Dado o elevado número de acidentes escorpiônicos registrados na cidade de Ponta Grossa e sua importância para a saúde pública, este estudo teve como objetivos; verificar a distribuição geográfica dos acidentes e reclamações de ocorrência de escorpiões; avaliar o conhecimento da população a respeito desse agravo; identificar as espécies ocorrentes na área urbana; realizar ação educativo-preventiva junto à comunidade escolar e ampliar o serviço de identificação. Foram utilizados dados referentes aos acidentes e reclamações ocorridos no ano de 2007. As coordenadas geográficas dos endereços correspondentes aos registros foram coletadas, digitalizadas e espacializadas, resultando em um cartograma georreferenciado. $\mathrm{O}$ interpolador não paramétrico Kernel foi utilizado para estimar o número de eventos por unidade de área. A aplicação de um inquérito populacional ocorreu no bairro Colônia Dona Luiza, devido a este ter apresentado o maior coeficiente de incidência para o período. Os dados coletados indicaram que $64,8 \%$ dos moradores 
já haviam encontrado escorpião, 29,6\% tinham sofrido acidente e $25,4 \%$ buscaram atendimento em uma unidade de saúde. Os exemplares capturados na busca ativa somados aos recolhidos e/ou encaminhados pela população para identificação revelaram a presença das espécies Tityus bahiensis e T. costatus, além do gênero Bothriurus. A realização de uma ação educativa para alunos da Educação Básica da rede pública de ensino oportunizoulhes obter conhecimento sobre a morfobiologia dos escorpiões e os principais riscos à saúde humana decorrentes da proliferação de aracnídeos nas áreas urbanas. Os resultados deste estudo poderão contribuir para ações que visem a prevenção de novos acidentes no município e na região.

Palavras-chave: Escorpiões. Saúde pública. Epidemiologia.

\begin{abstract}
Given the high number of accidents involving scorpions registered in the city of Ponta Grossa and its importance to public health, this study aimed to determine the geographic distribution of accidents and complaints of occurrence of scorpions; assess the population knowledge about this hazard, identifying the species found in the urban areas; conduct educational-preventive action with the school community and expand the identification service. We used data on accidents and complaints occurred in 2007. The geographic coordinates of addresses corresponding to the records were collected, scanned and spatialized resulting in a georeferenced cartogram. The interpolator nonparametric Kernel was used to estimate the number of events per unit area. The application of a population survey occurred in the neighborhood Colonia Dona Luiza, because it has shown the highest incidence rate for the period. The collected data indicated that $64.8 \%$ of the residents had already found a scorpion, 29.6\% had suffered an accident and 25.4\% sought care at a health facility. The specimens captured in active search added to the collected and/or submitted by the population for identification revealed the presence of Tityus bahiensis and T. costatus species, as well as the Bothriurus gender. The realization of an educational action for Basic Education students of public schools provided them the opportunity to obtain knowledge about the morphobiology of scorpions and the main risks to human health arising from the proliferation of arachnids in urban areas. The results of this study may contribute to actions aimed at preventing further accidents in the city and the region.
\end{abstract}

Keywords: Scorpions. Public health. Epidemiology.

\section{INTRODUÇÃO}

O escorpionismo é o quadro do envenenamento humano desencadeado pela inoculação do veneno escorpiônico, consequência dos acidentes com esses animais (BRASIL, 2009a). Os acidentes escorpiônicos são importantes em virtude da grande frequência com que ocorrem e da sua potencial gravidade (BRASIL, 2001), sendo considerado um problema de saúde pública devido à elevada incidência em várias regiões do país, com mais de 36.000 casos notificados em 2006 (BRASIL, 2009b).

Das cerca de 1.600 espécies conhecidas no mundo, apenas 25 podem causar acidente mortal. No Brasil, as principais espécies que causam acidentes graves são: Tityus serrulatus Lutz \& Mello, 1922, Tityus bahiensis
(Perty, 1933), Tityus stigmurus Thorell, 1877 e Tityus paraensis Kraepelin, 1896 (Tityus obscurus (Gervais, 1843)). Também foram registrados acidentes causados por outras espécies do gênero Tityus, porém sua incidência e gravidade são menores (CANDIDO, 2009).

A alta capacidade de colonização em ambientes urbanos deve-se, além das modificações ambientais, também às características reprodutivas dos escorpiões: são animais vivíparos, o período de gestação é curto, adquirem uma prole numerosa e apresentam cuidado maternal. A espécie T. serrulatus possui ainda capacidade de reproduzir-se por partenogênese, o que lhes confere uma fácil dispersão, adaptação e proliferação (BRASIL, 2009b). 
Os acidentes que em algumas cidades brasileiras têm se tornado um problema de saúde pública, pela sua frequência e/ou gravidade, ocorrem mais comumente nos períodos quentes e chuvosos, em função do desalojamento dos escorpiões de seus abrigos ocasionados pela água da chuva e da sua maior movimentação em busca de alimento (principalmente insetos) (BARBOSA et al., 2012; CAMPOLINA, 2006).

Entre 2001 e 2010, houve 1.209 acidentes causados por escorpiões na cidade de Ponta Grossa, com o maior número de registros ocorrido no ano de 2007 (SILVA, 2011). Apesar do elevado número de acidentes escorpiônicos registrados, ainda são insuficientes os estudos que abordam aspectos mais gerais desse agravo na cidade. Dos trabalhos realizados, destacam-se o de Nodari et al. (2006), que avaliaram parâmetros demográficos, espaciais e temporais de acidentes ocorridos na área de abrangência da $3^{\mathrm{a}}$ Regional de Saúde do Paraná - $3^{\mathrm{a}} \mathrm{RS} / \mathrm{PR}$, que inclui 12 municípios, entre eles Ponta Grossa; e o de Kotviski e Barbola (2013), que detalharam a distribuição espacial e os fatores ambientais relacionados ao escorpionismo na cidade de Ponta Grossa. É importante salientar que a análise dos acidentes causados por animais peçonhentos contribui amplamente para as ações de vigilância em saúde, permitindo elaborar estratégias de controle e programas de prevenção de acidentes (BARBOSA; MEDEIRO; COSTA, 2015).

Assim sendo, o presente trabalho visou aprofundar os conhecimentos acerca do escorpionismo em Ponta Grossa, tendo como objetivos específicos: 1) verificar a distribuição geográfica dos registros de acidentes e encontro de escorpiões nos imóveis; 2) avaliar os conhecimentos da população a respeito dos riscos desse agravo; 3 ) identificar as espécies ocorrentes na área urbana e conhecer o índice de infestação domiciliar de escorpiões; 4) realizar ação educativo-preventiva junto à comunidade escolar sobre esse tema; e 5) ampliar o serviço de identificação dos escorpiões depositados na Coleção de Artrópodes dos Campos Gerais do Paraná (DEBIO/UEPG).

\section{MATERIAL E MÉTODOS \\ Caracterização da área}

Ponta Grossa está localizada no Segundo Planalto Paranaense e na Mesorregião Centro-Oriental do Estado (Figura 1). Apresenta uma população de 311.611 habitantes (IBGE, 2010) e é considerada a cidade polo de uma das regiões mais populosas do Estado, os Campos Gerais do Paraná. O clima é do tipo $\mathrm{Cfb}$ - Subtropical Úmido Mesotérmico, com temperatura média anual entre $17^{\circ}$ e $18^{\circ} \mathrm{C}$ e com maior concentração de chuvas nos meses de verão (PMPG, 2006).

Figura 1 - Localização do município de Ponta Grossa no estado do Paraná e no Brasil

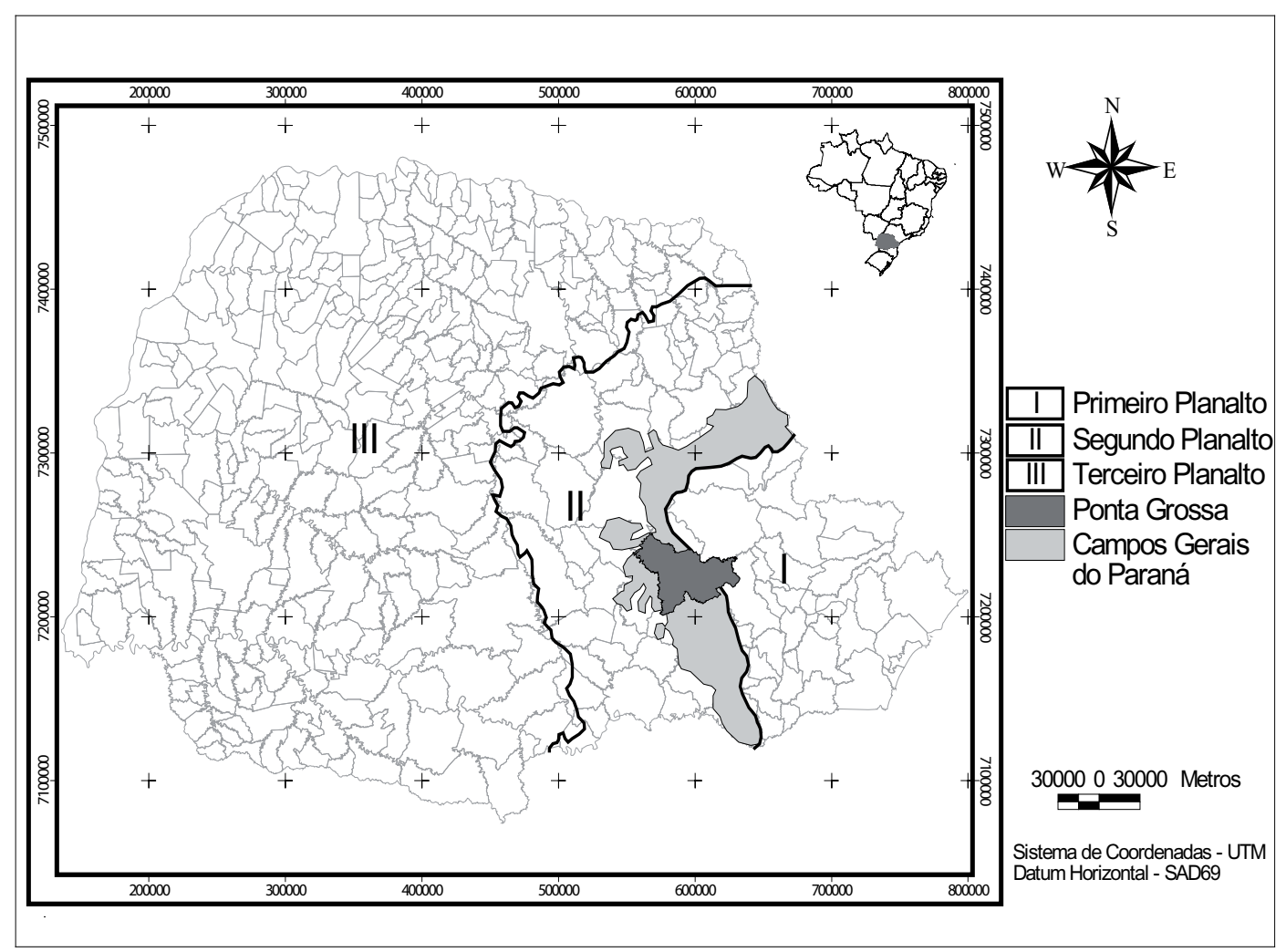




\section{Procedimentos metodológicos}

Esta pesquisa foi autorizada pelo Comitê de Ética em Pesquisa envolvendo Seres Humanos (COEP), da Universidade Estadual de Ponta Grossa, pelo Parecer de $n^{0} 58 / 08$ e pelo Processo $n^{\circ} 13240 / 08$.

Os dados utilizados correspondem aos registros de acidentes e de reclamações de ocorrência de escorpiões no município, no período de janeiro a dezembro de 2007. Os 168 registros de acidentes, contidos no Sistema de Informação de Agravos de Notificação (SINAN) foram disponibilizados pela Secretaria de Estado da Saúde do Paraná (SESA/PR), por intermédio da $3^{\mathrm{a}} \mathrm{RS} / \mathrm{PR}$. Já os 15 registros de reclamação de ocorrência de escorpiões nos imóveis foram disponibilizados pela Gerência de Controle de Zoonoses da Prefeitura Municipal de Ponta Grossa (GCZ/PMPG).

\section{Distribuição geográfica}

Visando conhecer a distribuição geográfica das ocorrências nos bairros, os registros de acidentes e de reclamações de escorpiões referentes ao período analisado foram georreferenciados. Entre julho e outubro de 2008, as coordenadas geográficas dos endereços correspondentes às notificações de 2007 foram coletadas in loco por meio do receptor de navegação - Global Positioning System (GPS) (marca GARMIN, modelo GPSMAP - 60CSx). Os dados foram inseridos em uma tabela, adequados para o Sistema de Projeção Universal Transversal de Mercator (UTM) e importados para o programa de Sistemas de Informação Geográfica (SIG) - ArcView 3.2a., onde foram espacializados.

Com a espacialização pontual dos dados, realizou-se no SIG a estimativa não paramétrica de densidade de probabilidade - densidade Kernel, ou seja, interpolaram-se os pontos com o estimador Kernel, o qual possibilita verificar a intensidade de eventos (acidentes e ou reclamações). De forma simplificada, o estimador Kernel consiste em estimar o número esperado de eventos por unidade de área. De acordo com Gatrell e Bailey (1996), esse estimador foi originalmente desenvolvido para obter uma estimativa de densidade de probabilidade tanto univariada como multivariada de uma amostra observada, pois estimar a intensidade de um padrão de pontos é como estimar uma densidade de probabilidade bivariada. Assim sendo, em sentido contrário, pode-se adaptar a estimativa bivariada de Kernel para se obter uma estimativa de intensidade do padrão de pontos (TERUIYA, 1999).

Figura 2 - Bairros do perímetro urbano de Ponta Grossa, PR

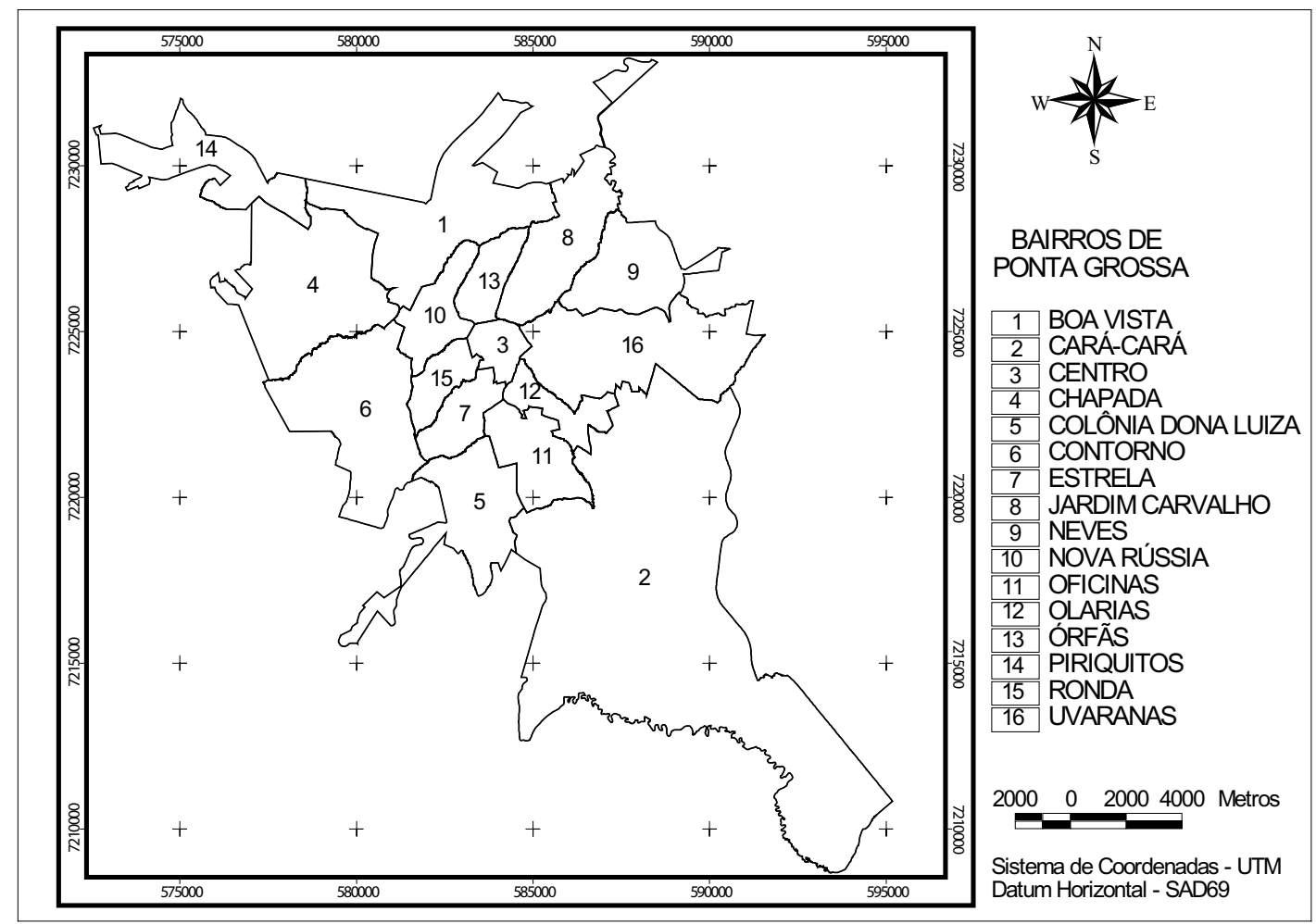




\section{Inquérito populacional}

A fim de avaliar os conhecimentos da comunidade a respeito do escorpionismo, foi realizado um inquérito populacional em agosto de 2009 para investigação das condições sanitárias domiciliares, da frequência de encontro de escorpiões no intra ou peridomicílio, e, em caso de acidentes, qual o procedimento utilizado pelo acidentado. O bairro escolhido para aplicação do questionário foi aquele que apresentou o maior coeficiente de incidência (C.I.), calculado para 15 dos 16 bairros da cidade onde houve registros (Figura 2), sendo o C.I. = $\mathrm{n}^{\mathrm{o}}$ de casos de uma doença ocorrentes em determinada comunidade em certo período de tempo $/ \mathrm{n}^{\circ}$ de pessoas expostas ao risco de adquirir a doença no referido período x $10^{n}$ (ROUQUAYROL; ALMEIDA FILHO, 1999). Foram utilizados dados do Censo 2010 por serem mais próximos do ano de análise (MAZER, 2011).

\section{Busca ativa}

Objetivando a identificação das espécies de escorpiões ocorrentes na área urbana, entre janeiro e fevereiro de 2010 , foram realizadas buscas ativas diurnas nos 15 bairros da cidade onde houve registros. Os locais de vistoria foram determinados com base na distribuição espacial dos registros, priorizando os locais com maior probabilidade de encontro desses animais. O número de imóveis vistoriados variou conforme o número desses registros, a proximidade dos endereços onde houve notificação e a existência de terrenos baldios e/ou residências com permissão de vistoria.

O índice de infestação domiciliar (IID = número de unidades domiciliares positivas / número de unidades domiciliares pesquisadas ou sob vigilância $\mathrm{x} 100$ ) foi calculado para avaliar a real situação da cidade (BRASIL, 2009b).

\section{Ação educativa}

Buscando orientar a comunidade escolar sobre os riscos da proliferação de artrópodes peçonhentos para a saúde humana, realizou-se uma ação educativa em maio de 2010 para alunos do $9^{\circ}$ ano da Escola Básica, em que foi abordado o tema "escorpionismo". No material didático elaborado, constaram dinâmicas e jogos pedagógicos, exemplares fixados, imagens e informações apresentadas em multimídia. Durante a atividade foram aplicados dois questionários: (1) verificação dos conhecimentos prévios dos alunos sobre o tema, em que foram abordadas questões a respeito da frequência de encontro desses animais, o ambiente onde vivem, do que se alimentam e quais as medidas para prevenção de acidentes; e (2) avaliação da ação educativa, com a verificação de quais informações que despertaram mais interesse dos alunos e quais os conhecimentos adquiridos, a partir dessa apresentação.

\section{Recolhimento e/ou recebimento}

Trata-se de uma atividade contínua que tem por objetivo ampliar o serviço de identificação dos exemplares de artrópodes encaminhados ao Laboratório de Conservação e Biodiversidade da UEPG pela comunidade em geral.

\section{Resultados e Discussão \\ Distribuição geográfica}

Ao todo, foram georreferenciadas 144 notificações de acidentes e 14 de ocorrência de escorpiões que possuíam logradouro identificável. A espacialização destas resultou em um cartograma georreferenciado de todos os registros de 2007, permitindo, por meio da sua visualização, a análise pontual das áreas com registros na cidade e a tendência de uma distribuição agregada (Figura 3 ).

A Figura 4 exibe os dados e a interpolação pelo estimador Kernel. À esquerda, os limites dos bairros e a intensidade de ocorrência; e, à direita, a intensidade de ocorrência com os limites dos bairros e, ainda, pontualmente, os acidentes. Nessa figura, pode-se verificar a intensidade de incidência baixa, média e alta. O estimador mostra os bairros Colônia Dona Luiza, Olarias e Jardim Carvalho como os que apresentaram maior intensidade de acidentes registrados.

De acordo com Barbosa et al. (2003), características como a falta de saneamento básico nos arredores das residências dos acidentados, a proximidade com construções inacabadas, coletas irregulares de lixo e acúmulo de entulhos contribuem para a proliferação de escorpiões e, consequentemente, no aumento do número de acidentes domiciliares. As maiores incidências de acidentes nos bairros apontados neste estudo podem estar relacionadas, ainda, à proximidade de margens de rio, de córregos e de riachos, de galerias de águas pluviais, de canais, de galerias de esgoto e de bocas de lobo, consideradas também focos de ocorrência de escorpiões (BRASIL, 2009b). Tais fatores precisam 
ser melhor investigados, mas, de acordo com Nunes, Bevilacqua e Jardim (2000), as características do ambiente associadas à forma de ocupação do espaço urbano oferecem condições ambientais favoráveis para o abrigo, a alimentação e a proliferação de escorpiões.

Figura 3 - Espacialização dos registros de acidente e de reclamação de ocorrências de escorpiões na cidade de Ponta Grossa, em 2007

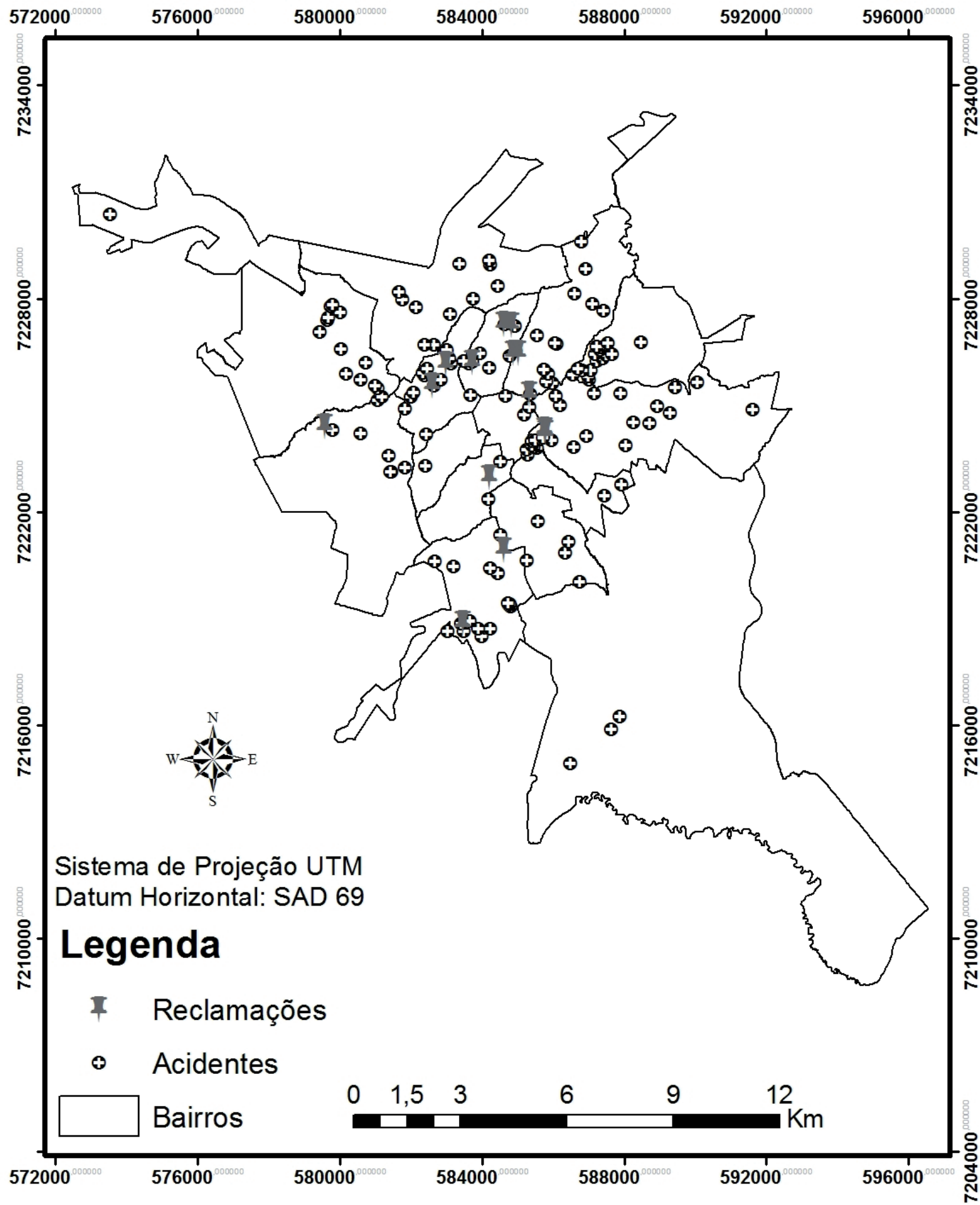


Figura 4 - Estimador Kernel de intensidade de ocorrência dos acidentes com escorpiões na área urbana de Ponta Grossa, em 2007

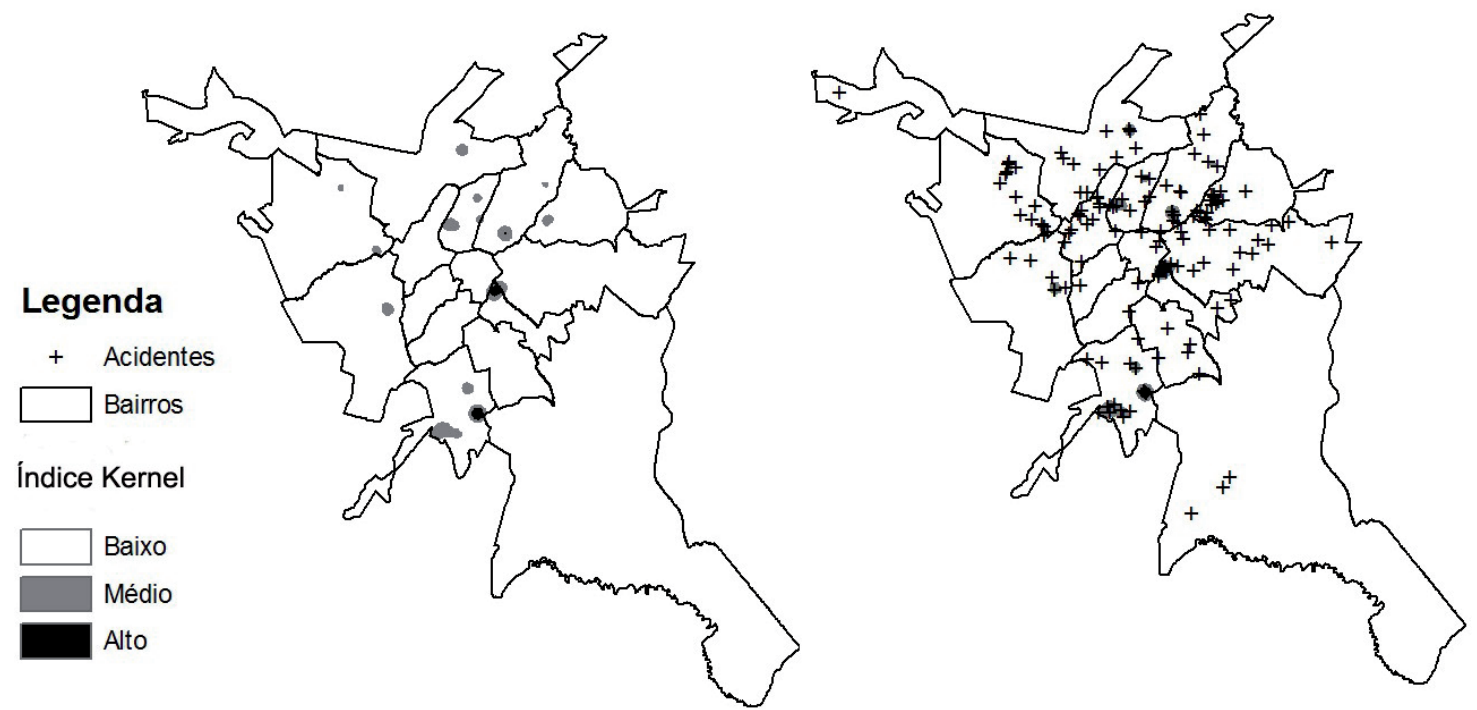

Tabela 1 - Número de acidentes, por bairro, causados por escorpiões na cidade de Ponta Grossa em 2007 e respectivos valores do coeficiente de incidência (C.I.)

\begin{tabular}{c|c|c|c}
\hline BAIRROS & $\mathbf{N}^{\mathbf{0}}$ DE ACIDENTES & $\mathbf{N}^{\mathbf{0}}$ DE HAB. & $\mathbf{C . I}_{\mathbf{~}}(\mathbf{1 0} \mathbf{5})$ \\
\hline COLONIA DONA LUIZA & 14 & 16,639 & 84,14 \\
\hline OLARIAS & 7 & 8,545 & 81,92 \\
\hline NEVES & 17 & 22,211 & 76,54 \\
\hline NOVA RÚSSIA & 15 & 19,656 & 76,31 \\
\hline CHAPADA & 17 & 23,166 & 73,38 \\
\hline JARDIM CARVALHO & 16 & 22,393 & 71,45 \\
\hline ORFÃS & 9 & 13,107 & 68,67 \\
\hline UVARANAS & 18 & 44,450 & 40,49 \\
\hline BOA VISTA & 9 & 24,968 & 36,05 \\
\hline OFICINAS & 7 & 20,414 & 34,29 \\
\hline RONDA & 3 & 9,229 & 32,51 \\
\hline CENTRO & 3 & 12,325 & 24,34 \\
\hline CONTORNO & 5 & 28,386 & 17,61 \\
\hline ESTRELA & 1 & 7,548 & 13,25 \\
\hline CARÁ CARÁ & 3 & 24,779 & 12,11 \\
\hline PIRIQUITOS & 0 & 5,417 & 0,00 \\
\hline TOTAL & 144 & & \\
\hline
\end{tabular}

\section{Inquérito populacional}

O coeficiente de incidência encontrado para o município, considerando o número de habitantes da cidade (311.611) e todos os acidentes notificados em 2007 (144), foi de 46,2 casos por 100 mil habitantes. Esse valor é mais que o dobro da incidência nacional e quase sete vezes maior que a estadual (BRASIL, 2016).
A Tabela 1 mostra o número de acidentes e os valores de C.I. calculados para os diferentes bairros da cidade. O inquérito populacional foi aplicado no bairro Colônia Dona Luiza, tendo em vista que este apresentou o maior C.I., o que também foi confirmado pelo estimador Kernel. Nesse bairro, foram efetuados os inquéritos nos endereços referentes a 14 registros de acidentes e dois registros de ocorrência do animal, incluindo os cinco imóveis limítrofes em cada uma 
dessas unidades domiciliares. Dos 96 imóveis esperados para aplicação do inquérito populacional, quatro apresentaram um dos imóveis limítrofes conjugados e cinco imóveis se sobrepuseram, apresentando, assim, o mesmo endereço, representando, dessa forma, um real de 87 esperados para aplicação do questionário.

Ao longo de cinco dias, 87 imóveis foram visitados, sendo $77(88,5 \%)$ residências, quatro $(4,6 \%)$ terrenos baldios, duas (2,3\%) igrejas, dois $(2,3 \%)$ comércios, uma $(1,1 \%)$ escola e um $(1,1 \%)$ cemitério. Dos imóveis visitados, 71 participaram efetivamente da pesquisa, os demais estavam fechados (11), eram terrenos baldios (4) ou os moradores recusaram-se a participar (1). Entre os 71 participantes efetivos, 46 $(64,8 \%)$ já haviam encontrado escorpião em seus imóveis, e $25(35,2 \%)$ nunca haviam encontrado.

Em 21 (29,6\%) imóveis houve acidente. Destes, $18(85,7 \%)$ moradores buscaram atendimento em uma unidade de saúde. Esses valores são bem superiores aos encontrados por Amorim et al. (2003), no bairro de Areal, em Salvador, BA, em que 6\% dos indivíduos entrevistados referiram ter sido picados por escorpião (dos quais $42 \%$ procuraram algum serviço de saúde), e, também, por Barbosa et al. (2003) que encontraram percentuais de acidentados de 19,52\%, 16,65\% e $8,78 \%$, respectivamente, para os bairros Vale das Pedrinhas, Nordeste de Amaralina e Cajazeiras, também em Salvador.

Por meio do inquérito, foi possível constatar acidentes não notificados pelos moradores do bairro em questão, o que demonstra que o número real de acidentes pode ser muito maior do que o registrado no período. Além disso, se considerarem-se as falhas inerentes no sistema de notificação, depara-se com a grande preocupação do subdimensionamento do agravo, ao basear-se somente nos registros do SINAN.

\section{Busca ativa}

A ação de busca ativa permitiu a vistoria de 45 imóveis: 22 deles residências (48,9\%), 16 terrenos (35,6\%) e sete outros (15,6\%). Houve coleta em nove (20\%) imóveis, sendo que dos 27 exemplares capturados, 14 (51,9\%) em madeiras, $6(22,2 \%)$ em caixa de esgoto, $4(14,8 \%)$ em entulhos, 2 (7,4\%) em tijolos e $1(3,7 \%)$ em outro. Dos indivíduos capturados, $15(55,6 \%)$ pertencem à espécie Tityus costatus, 10 (37\%) à T. bahiensis e dois $(7,4 \%)$ ao gênero Bothriurus.
Ponta Grossa apresentou um índice de infestação domiciliar (IID) correspondente a 20\% (ou seja, entre $1-25 \%$ ), sendo classificada de infestação/dispersão média, uma vez que valores entre 26 e 50\% correspondem à infestação alta, e acima de $50 \%$ são considerados de altíssima infestação (BRASIL, 2009b). Apesar dos locais de vistoria terem sido escolhidos considerando-se a maior probabilidade de encontro desses animais, se fossem ampliadas as áreas de vistoria e incluído o método de captura noturna com emprego de luz ultravioleta, é possível que o valor do IID fosse maior do que o obtido. Brites-Neto e Brasil (2012) observaram um aumento de $114 \%$ na média anual de captura noturna (com emprego de luz ultravioleta), quando comparado à coleta mecânica diurna.

\section{Ação educativa}

Quanto à ação educativa, os 26 alunos presentes responderam aos questionários. Na verificação dos conhecimentos prévios a respeito do tema, $17(65,4 \%)$ escolares disseram já ter encontrado escorpiões em suas residências e houve acidente (picadas) em cinco $(19,2 \%)$ delas.

A minoria revelou falta de informação em relação aos hábitos de vida dos escorpiões, os riscos de acidentes e as medidas preventivas desse agravo: $7(26,9 \%)$ não responderam corretamente sobre qual o principal alimento dos escorpiões e 9 (34,6\%) não souberam informar a medida mais eficaz para evitar a presença desses aracnídeos nas casas.

No pós-teste, $15(57,7 \%)$ alunos afirmaram que saberão comentar o assunto com seus colegas, 13 (50\%) com seus irmãos, $21(80,8 \%)$ com seus pais e $12(46,2 \%)$ com outras pessoas; $23(88,5 \%)$ irão aproveitar o que aprenderam sobre o tema, conforme a transcrição de duas respostas selecionadas aleatoriamente para representar a opinião da turma sobre o que fariam se encontrassem um escorpião: “[...] eu ia tomar cuidado para não ser picado e, se fosse picado, passaria sabão e procuraria imediatamente um posto médico" (Aluno 9); "[...] iria colocar em um recipiente com tampa para fazer umas avaliações sobre ele" (Aluno 4). Quando indagados sobre quais as informações mais interessantes apresentadas durante a palestra, as principais respostas foram "prevenção" e "habitat", indicando que adquiriram conhecimento significativo sobre o ambiente onde vivem os escorpiões e quais 
as principais medidas para evitar acidentes por esses aracnídeos.

$\mathrm{Na}$ avaliação do impacto da ação educativa, 21 (80,8\%) alunos conceituaram-na como muito boa e 19 $(73,1 \%)$ deles afirmaram que gostariam de participar de outra atividade como esta. Com esses resultados, considera-se que os objetivos da ação foram atingidos, pois permitiram capacitar os alunos a reconhecer os escorpiões de interesse médico e os ambientes onde são encontrados, proporcionando-lhes condições para a prevenção de acidentes e a socialização desses conhecimentos. Assim, abordagens direcionadas ao tema escorpionismo representam uma boa estratégia de aprendizado, e a utilização de recursos lúdicos de fácil assimilação pode auxiliar na construção de conhecimento coletivo (SENNA-NUNES et al., 2004) e gerar resultados de aprendizagem superiores às clássicas aulas expositivas.

\section{Recolhimento e/ou recebimento}

A visitação nos bairros promoveu a sensibilização e a participação dos moradores, em uma atividade contínua, que visa o recolhimento e/ou recebimento de exemplares encaminhados pela população, a qual foi alertada a respeito dos riscos para a saúde que esses artrópodes podem trazer. A busca de estratégias integradas de informação, educação, comunicação em saúde e mobilização comunitária produz mudanças de comportamentos e práticas voltadas ao desenvolvimento de capacidades individuais e coletivas visando a formação do ser sadio (SENNA-NUNES et al., 2004).

Dos 65 escorpiões recolhidos e/ou recebidos entre 2007 e 2012, 48 (73,8\%) foram identificados como T. bahiensis, $14(21,5 \%)$ como T. costatus e três $(4,6 \%)$ como Bothriurus sp. Esses exemplares, após identificação, foram incorporados à Coleção de Artrópodes dos Campos Gerais do Paraná.

As espécies encontradas neste estudo foram as mesmas já identificadas pela SESA/PR para a cidade (FIGUEIREDO, 2011), não tendo sido encontrado nenhum exemplar de Tityus pintodarochai, 2005, espécie aparentemente mais silvestre, descrita a partir de um indivíduo coletado no Parque Estadual de Vila Velha, situado a cerca de $20 \mathrm{~km}$ do centro de Ponta Grossa (LOURENÇO, 2005).

Dentre as espécies encontradas, somente $T$. bahiensis é considerada de interesse em saúde pública e, embora o escorpião marrom tenha veneno menos tóxico do que o T. serrulatus, é responsável por muitos dos acidentes registrados, cujos pacientes necessitam, por vezes, de atendimento médico disponibilizado no serviço público (RAMIRES et al., 2011).

Pelo fato de nenhum exemplar de T. serrulatus ter sido identificado até setembro de 2012, acredita-se que esse realmente ainda não esteja presente na área urbana de Ponta Grossa, já que é uma espécie numerosa nas regiões em que habita, devido à sua alta capacidade de dispersão e forrageamento, ao seu elevado potencial reprodutivo e à grande reserva de populações (LOURENÇO et al., 1996). O que reforça a suspeita de Nodari et al. (2006), que salientaram que, apesar dos registros de ocorrência da espécie T. serrulatus por meio dos dados oficiais da $3^{\text {a }}$ RS/PR entre 2001 e 2004, existem dúvidas quanto a sua ocorrência na região. É possível que as informações referentes ao aracnídeo tenham sido notificadas sem confirmação da espécie do animal, ou apenas seguido relatos imprecisos dos pacientes.

\section{CONCLUSÃO}

A espacialização dos registros permitiu a visualização das áreas de infestação de escorpiões na cidade de Ponta Grossa (PR) que necessitam maior atenção por parte dos órgãos responsáveis pelo controle do escorpionismo. O estimador Kernel complementou as informações fornecidas pela espacialização pontual, pois mostrou a intensidade do acidente e, portanto, a dependência espacial dele, agregando à análise visual pontual a estimativa de ocorrência na área de estudo. Mediante a organização desses dados em SIG, foi possível adequar os dados para visualizações exploratórias adequadas, o que possibilitou a extração das informações relevantes, como a posição geográfica dos acidentes e as reclamações do evento do escorpionismo, bem como a intensidade dos eventos nos bairros da cidade de Ponta Grossa, fato este que culminou na intenção de futuras análises ambientais e epidemiológicas nos anos subsequentes.

A aplicação do inquérito populacional possibilitou avaliar o conhecimento da população local a respeito da problemática e evidenciou que a maioria dos entrevistados já encontrou escorpiões em suas residências e cerca de $30 \%$ deles sofreram acidentes, procurando a maioria dos acidentados atendimento em uma unidade de saúde. 
A busca ativa revelou que as espécies de escorpiões encontradas em Ponta Grossa são Tityus bahiensis, T. costatus e Bothriurus sp.

A ação educativa realizada para alunos da Escola Básica obteve resultados satisfatórios, visto que temas como escorpionismo são pouco abordados tanto no âmbito escolar quanto na comunidade em geral.

Ações públicas visando principalmente esclarecer sobre os riscos de acidentes e, com isso, promover mudanças de hábitos e comportamentos da população devem ser constantes (BARBOSA et al., 2012). Medidas implementadas no âmbito municipal podem desempenhar influência direta na redução do número de acidentes escorpiônicos (BRITES-NETO; BRASIL, 2012). Assim sendo, acredita-se que os resultados deste estudo poderão contribuir para ações que visem a redução e a prevenção de novos acidentes em Ponta Grossa e na região.

\section{AGRADECIMENTOS}

Os autores agradecem à Divisão de Vigilância de Zoonoses e Intoxicação da SESA/PR e à Gerência de Controle de Zoonoses da PMPG, pela disponibilização dos dados; à Fundação Araucária, pelas bolsas de iniciação científica concedidas; ao Sr. Mauro Carvalho Leal e Sr. Edson dos Santos, pelo apoio na obtenção das coordenadas geográficas; aos geógrafos Rodrigo Antonio La Scalea e Emelly Cristine Kozowski, pelo auxílio na etapa de espacialização; aos biólogos Solange Burgardt e Alexandre Camargo de Azevedo, pela contribuição na etapa de busca ativa; ao Departamento de Geociências da UEPG pela disponibilização da estrutura do Laboratório de Geotecnologias; à Direção do Colégio Estadual Polivalente, Ponta Grossa, PR.

\section{REFERÊNCIAS}

AMORIM, A. M.; CARVALHO, F. M.; LIRA-DA-SILVA, R. M.; BRAZIL, T. K. Acidentes por escorpião em uma área do Nordeste de Amaralina, Salvador, Bahia, Brasil. Revista da Sociedade Brasileira de Medicina Tropical, v. 26, n. 1, p. 51-56, 2003.

BARBOSA, M. G. R.; BAVIA, M. E.; SILVA, C. E. P.; BARBOSA, F. R. Aspectos epidemiológicos dos acidentes escorpiônicos em Salvador, Bahia, Brasil. Ciência Animal Brasileira, v. 4, n. 2, p. 155-162, 2003.

BARBOSA, A. D; MAGALHÃES, D. F.; SILVA, J. A.; SILVA, M. X.; CARDOSO, M. F. E. C.; MENESES, J. N. C.; CUNHA, M. C. M. Caracterização dos acidentes escorpiônicos em Belo Horizonte, Minas Gerais, Brasil,
2005 a 2009. Cad. Saúde Pública, v. 28, n. 9, p. 1785-1789, 2012.

BARBOSA, I. R.; MEDEIROS, W. R.; COSTA, I. C. C. DISTRIBUIÇÃO ESPACIAL DOS ACIDENTES POR ANIMAIS PEÇONHENTOS NO ESTADO DO RIO GRANDE DO NORTE-BRASIL NO PERÍODO DE 20012010. Caminhos de Geografia, v. 16, n. 53, p. 55-64, 2015.

BRASIL. Ministério da Saúde. Incidência (100.000 hab) de casos de acidentes por escorpiões. Brasil, Grandes Regiões e Unidades Federadas. 2000 a 2013*. Disponível em: $\quad<h t t p: / / u . s a u d e . g o v . b r / i m a g e s / p d f / 2014 / j u l h o / 10 /$ Tabela-10---INCIDENCIA-CASOS---escorpiao--2000-a-2013---21-05-2013.pdf>. Acesso em: 04 mai. 2016.

BRASIL. Ministério da Saúde. Fundação Nacional de Saúde. Manual de diagnóstico e tratamento de acidentes por animais peçonhentos. 2. ed. Brasília: Fundação Nacional de Saúde, 2001. 120 p.

BRASIL. Ministério da Saúde. Secretaria de Atenção Básica. Departamento de Atenção Básica. Vigilância em saúde: zoonoses. Brasília: Ministério da Saúde, 2009a. 224 p.

BRASIL. Ministério da Saúde. Secretaria de Vigilância em Saúde. Departamento de Vigilância Epidemiológica. Manual de Controle de Escorpiões. Brasília: Ministério da Saúde, 2009b. 70 p.

BRITES-NETO, J.; BRASIL, J. Estratégias de controle do escorpionismo no município de Americana, SP. BEPA, v. 9, n. 101, p. 4-15, 2012.

CAMPOLINA, D. Georreferenciamento e estudo clínicoepidemiológico dos acidentes escorpiônicos atendidos em Belo Horizonte, no Serviço de Toxicologia de Minas Gerais. 2006, 152 f. Dissertação (Mestrado em Infectologia e Medicina Tropical) - Universidade Federal de Minas Gerais, Belo Horizonte, 2006.

CANDIDO, D. M. Escorpiões (Scorpions, Alacranes). 2009, 17 f. Apostila Aracnídeos. Laboratório de Artrópodes. Instituto Butantan. São Paulo.

FIGUEIREDO, W. Espécies de escorpiões encontradas no município de Ponta Grossa. [Mensagem profissional]. Mensagem recebida por: <bianca_kotviski@hotmail.com> em 31 ago. 2011.

GATRELL, A. C.; BAILEY, T. C. Interactive Spatial Data Analysis in Medical Geography. Social Science \& Medicine, v. 42, n. 6, p. 843-855, 1996.

IBGE - INSTITUTO BRASILEIRO DE GEOGRAFIA E ESTATÍSTICA. População da cidade de Ponta Grossa em 2010. Disponível em: <http://cod.ibge.gov.br/6N1>.

KOTVISKI, B. M.; BARBOLA, I. F. Aspectos espaciais do escorpionismo na cidade de Ponta Grossa, Paraná, Brasil. Cad. Saúde Pública, v. 29, n. 9, p. 1843-1858, 2013. 
LOURENÇO, W. R.; CLOUDSLEY-THOMPSON, J. L.; CUELLAR, O.; VON EICKSTEDT, V. R. D.; BARRAVIERA, B.; KNOX, M. B. The evolution of scorpionism in Brazil in recent years. Journal of Venomous Animals and Toxins, v. 2, n. 2, 1996.

LOURENÇO, W. R. Description of a new species of Tityus (Scorpiones, Buthidae) from the Parque Estadual de Vila Velha in the State of Paraná (Brazil). Acta Biol. Par., Curitiba, v. 34, n. 1, 2, 3, 4, p. 15-26, 2005.

MAZER, H. Tabela 608 - População residente, por situação do domicílio e sexo - Sinopse [Mensagem profissional]. Mensagem recebida por: <bianca_kotviski@ hotmail.com> em 18 out. 2011.

NODARI, F. R.; LEITE, M. L.; NASCIMENTO, E. Aspectos demográficos, espaciais e temporais dos acidentes escorpiônicos ocorridos na área de abrangência da $3^{\mathrm{a}}$. Regional de Saúde - Ponta Grossa, PR, no período de 2001 a 2004. Publicatio UEPG Ciências Biológicas e da Saúde, Ponta Grossa, v. 12, n. 1, p. 15-26, mar. 2006.

NUNES, C. S., BEVILACQUA P. D., JARDIM C. C. G. Aspectos demográficos e espaciais dos acidentes escorpiônicos no Distrito Sanitário Noroeste, Município de Belo Horizonte, Minas Gerais, 1993 a 1996. Cad. Saúde Pública, v. 16, n. 1, p. 213-223, 2000.

PMPG - PREFEITURA MUNICIPAL DE PONTA GROSSA. Plano Diretor Participativo. pdf. Ponta Grossa, 2006. Disponível em: <http://www.pg.pr.gov.br/ planodiretor>.

RAMIRES, E. N.; NAVARRO-SILVA, M. A.; MARQUES, F. A. Chemical Control of Spiders and Scorpions in Urban Areas. In: STOYTCHEVA,M. Pesticides in the Modern World - Pests Control and Pesticides Exposure and Toxicity Assessment. Croácia: InTech, 2011, cap. 24. p. 553-600.

ROUQUAYROL, M. Z.; ALMEIDA FILHO, N. Epidemiologia \& Saúde. 5. ed. Rio de Janeiro: UNIFOR, 1999. 327p.

SENNA-NUNES, M. S.; SILVA, C. C. M.; BARBOSA, G. M. S.; BERNARDINO, H. Enteroparasitoses em Comunidade Escolar de Instituições de Ensino Circunvizinhas à Universidade Iguaçu, Município de Nova Iguaçu, RJ. In: $2^{\circ}$ Congresso Brasileiro de Extensão Universitária, 2004, Belo Horizonte. Anais eletrônicos. Belo Horizonte: Universidade Federal de Minas Gerais, 2004. Disponível em: <http://www.ufmg.br/congrext/Saude/Saude79.pdf $>$.

SILVA, E. M. Frequência de acidentes por escorpiões por município e Regional de Saúde no Paraná entre 2001 e 2010. [Mensagem profissional]. Mensagem recebida por: <bianca_kotviski@hotmail.com> em 11 abr. 2011.

TERUIYA, R. K. Análise exploratória de dados socioeconômicos do município do Rio de Janeiro.
1999, 23 f. Trabalho apresentado como requisito parcial para aprovação na disciplina SER-301 Análise Espacial - Instituto Nacional de Pesquisas Espaciais, São José dos Campos, 1999. 